This item was submitted to Loughborough's Research Repository by the author.

Items in Figshare are protected by copyright, with all rights reserved, unless otherwise indicated.

\title{
Mammography workstation design: effect on mammographer behaviour and the risk of musculoskeletal disorders
}

PLEASE CITE THE PUBLISHED VERSION

PUBLISHER

(C) 2008 Society of Photo-Optical Instrumentation Engineers

VERSION

VoR (Version of Record)

LICENCE

CC BY-NC-ND 4.0

\section{REPOSITORY RECORD}

Taylor-Phillips, Sian, Matthew G. Wallis, and Alastair G. Gale. 2019. "Mammography Workstation Design: Effect on Mammographer Behaviour and the Risk of Musculoskeletal Disorders". figshare. https://hdl.handle.net/2134/6300. 
This item was submitted to Loughborough's Institutional Repository (https://dspace.lboro.ac.uk/) by the author and is made available under the following Creative Commons Licence conditions.

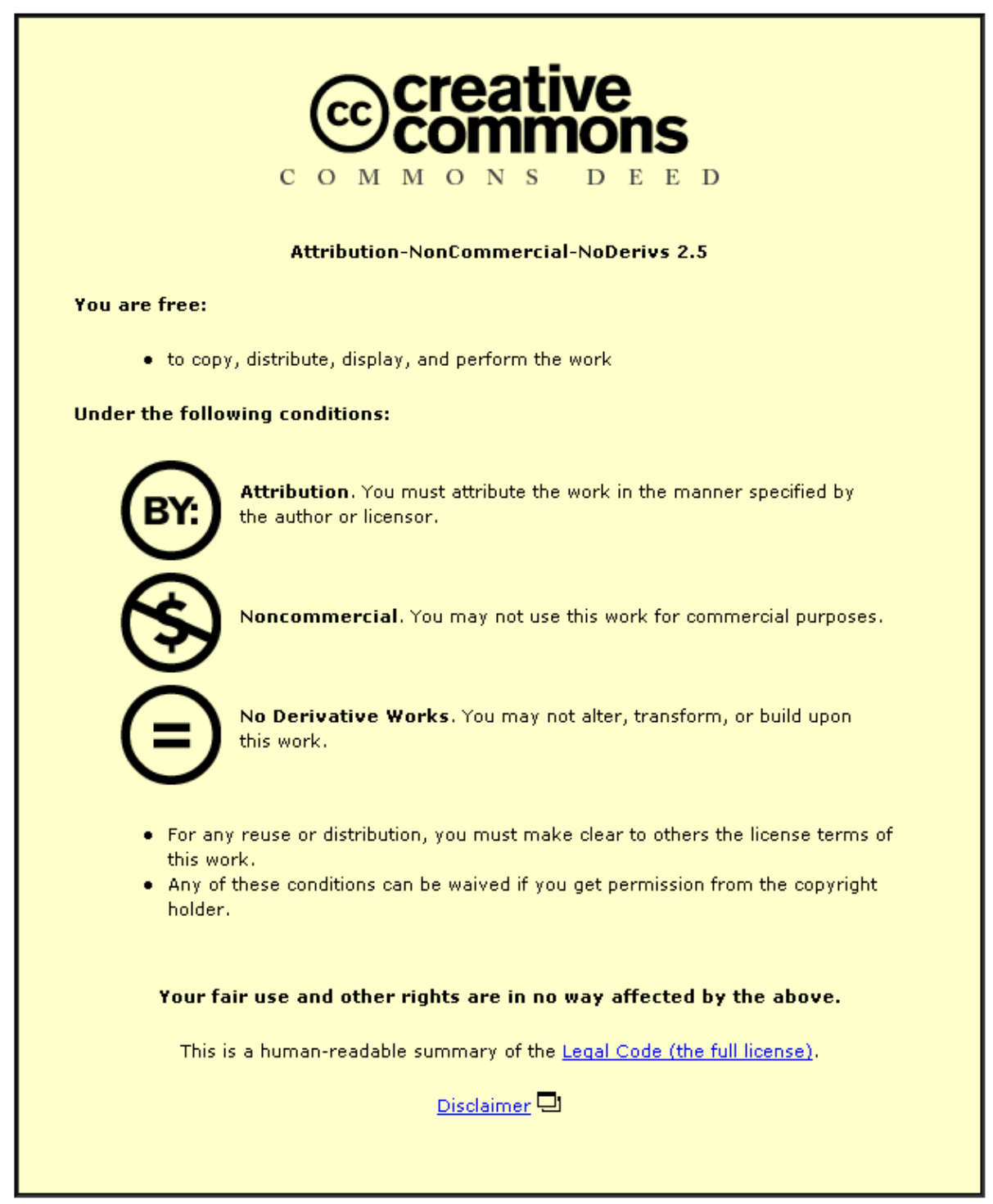

For the full text of this licence, please go to: http://creativecommons.org/licenses/by-nc-nd/2.5/ 


\title{
Mammography Workstation Design: Effect on Mammographer Behaviour and the Risk of Musculoskeletal Disorders
}

\author{
S.Taylor-Phillips ${ }^{1}$ \\ M.G. Wallis ${ }^{2}$ \\ A.G.Gale ${ }^{1}$ \\ ${ }^{1}$ Applied Vision Research Centre, Loughborough University, UK \\ ${ }^{2}$ Cambridge Breast Unit, Addenbrookes Hospital, Cambridge, UK
}

\begin{abstract}
In the UK Breast Screening Programme there is a growing transition from film to digital mammography, and consequently a change in mammography workstation ergonomics. This paper investigates the effect of the change for radiologists including their comfort, likelihood of developing musculoskeletal disorders (MSD's), and work practices. Three workstations types were investigated: one with all film mammograms; one with digital mammograms alongside film mammograms from the previous screening round, and one with digital mammograms alongside digitised film mammograms from the previous screening round. Mammographers were video-taped whilst conducting work sessions at each of the workstations. Event based Rapid Upper Limb Assessment (RULA) postural analysis showed no overall increase in MSD risk level in the switch from the film to digital workstation. Average number of visual glances at the prior mammograms per case measured by analysis of recorded video footage showed an increase if the prior mammograms were digitised, rather than displayed on a multi-viewer $(\mathrm{p}<.05)$. This finding has potential implications for mammographer performance in the transition to digital mammography in the UK.
\end{abstract}

Key words: Digital mammography, digitisation, previous mammogram, musculoskeletal disorders, workstation, eye movements.

\section{INTRODUCTION}

In the UK the National Health Service provides a Breast Screening Programme (NHSBSP) where every woman aged 5070 (to be extended to women aged 47 to 73 years by 2012) is invited for breast cancer screening every three years. Two views are taken of each breast: mediolateral-oblique (MLO); and cranio-caudal (CC). The mammograms from each woman screened are double read by either breast screening radiologists or appropriately qualified breast screening radiographers (advanced practitioners), for simplicity henceforth these will all be referred to as mammographers. For a woman attending breast screening for the first time (at the incident screening round) the mammographer's task is to examine the four mammograms taken and decide whether to recall the woman for further tests. At subsequent screening rounds for the same woman, the mammographer will have eight mammograms to examine, the four mammograms just taken plus the four mammograms from the screening round three years previously, henceforth these will be referred to as the current mammograms and the prior mammograms respectively. Mammographers can use comparisons between the current and prior mammograms to assist them in deciding whether to recall the woman for further assessment, for example looking at whether a mass has changed in size or shape, and whether microcalcifications were present for three years or have developed more recently. There are many scientific papers which show that using prior mammograms in addition to current mammograms improves mammographer performance ${ }^{1-3}$. Furthermore mammographers' performance is improved when prior mammograms are always present rather then present when requested by the mammographer ${ }^{4}$.

Currently less than ten breast screening centres in the NHSBSP use digital technology for screening, with the majority using film screen technology. Investigations have been underway in the UK about which digital mammography equipment is appropriate for use in the NHSBSP, and the role that Computer Aided Detection (CAD) may play. Single mammographers were asked to blindly reassess, with the assistance of $\mathrm{CAD}$, cases which had been assessed by double

Medical Imaging 2008: Image Perception, Observer Performance, and Technology Assessment, edited by Berkman Sahiner, David J. Manning, Proc. of SPIE Vol. 6917, 69171G, (2008)

1605-7422/08/\$18 - doi: 10.1117/12.770202

Proc. of SPIE Vol. 6917 69171G-1 
reading in the NHSBSP in 1996. It was found that single reader assessment with CAD increased both the cancer detection rate but also the recall rate versus the initial double reader assessment. A part of this effect may be due to changes in mammographer approach and performance since 1996, furthermore the mammograms used in the study were MLO view only which does not reflect current practice 5 . The CADET II study in the UK which compares use of double reader to single reader with CAD in the live screening programme are expected to be published in 2008 , and will be influential in determining the future direction of mammography reading in the UK. It is hoped that CAD can ease some of the potential workforce problems in the NHSBSP ${ }^{6}$. Therefore with this evidence collected it is likely that the majority of breast screening centres will start to employ digital technology for screening within the next few years.

When digital technology is first introduced to each breast screening centre there will be a period of transition, where the current mammograms are digital and the prior mammograms (taken three years previously) remain in film form. It is important that the performance of mammographers is not adversely affected both during and after this transition period. During the transition period there are three options for displaying the prior mammograms. Firstly a light box could be provided for mammographers to hang the prior mammograms when they feel it necessary. Secondly all of the prior mammograms could be hung on a multi-viewer placed alongside the digital display screen (a 'hybrid' workstation). Finally the prior mammograms could be digitised and displayed on the LCD screen alongside the current mammograms (a 'digital' workstation). In the Dutch screening programme all prior mammograms from one screening round previously will be digitised, in light of the evidence showing the importance of prior mammograms, and the difficulties for the mammographer in using a hybrid workstation ${ }^{7}$. Both the hybrid and film workstations are under consideration for use during the transition period in the UK Breast Screening Programme. At the hybrid workstation the mammographers do not have to request or hang the prior mammograms, which has been shown to decrease performance ${ }^{4}$, but they do have to turn their head to the multi-viewer and adapt to the different light levels. It is not known whether the effort involved in this type of regular body movement will result in mammographers using the prior mammograms less at this workstation. This data is crucial in deciding whether to digitise the prior mammograms.

Workstation ergonomics is important both during and after the transition to digital mammography, because mammographers' comfort and risk of developing musculoskeletal disorders are influenced by workstation design, and furthermore the design of the workstation can affect the mammographer's behaviour and consequently performance. There is evidence to suggest that decreasing ambient light levels and increasing monitor light levels improves mammographer performance ${ }^{8,9}$. In considering radiology room design various papers recommend implementing PACS workstations with parallel adjacent light boxes, angled adjacent light boxes, and light boxes above the workstation ${ }^{10-12}$. However this is for generic radiology workstations and would not meet the specific needs of screening mammography due to the importance of prior mammograms in this particular field.

This paper reports on an investigation into the average number of times per case that mammographers look at and visually examine the prior mammograms at the digital and hybrid workstations, alongside ergonomic considerations, to inform the debate about whether the prior mammograms should be digitised. Data about the film workstation is also provided for the purposes of making comparisons to the current situation.

\section{METHOD}

Three workstations were investigated: film; hybrid; and digital. The film workstation consisted of a backlit multi-viewer with both current film and prior film mammograms displayed together. Multi-viewer film workstations are currently used throughout the UK Breast Screening Programme. The hybrid workstation contained two LCD screens to display the current digital mammograms, and an adjacent backlit multi-viewer to display the prior film mammograms. This hybrid workstation represents the likely situation for the three years of transition from film to digital technology in the UK. The digital workstation has two LCD screens displaying the current digital mammograms alongside the digitised prior mammograms, which provides an alternative to the hybrid workstation for the transition period between film and digital technology. All three workstations were in fact part of the same workstation, with different aspects used, see figure 1. The multi-viewer was a Mammolux XL. The digital mammograms were acquired and displayed using the Sectra MicroDose system with EIZO Radiforce $54 \mathrm{~cm}$ LCD screens. Prior mammograms at the digital workstation were digitised using the Array 2905 Laser Film Digitiser set to $75 \mu \mathrm{m}$ standard. The hanging protocol for the multi-viewer used where the research was conducted was to display current mammograms on the top row and prior mammograms on the row below. The hybrid workstation displayed three hangings for the current mammograms on the LCD screen: all four 
digital current mammograms; the current MLO views; and the current CC views. The prior mammograms were hung on the multi-viewer with MLO views on the top level and CC on the bottom. The digital workstation displayed three hangings: the same hanging protocol as the film workstation; the current MLO views; and the current CC views. Ambient lighting was switched off at the workstation during the experiment, so the only light sources were the multiviewer (when switched on), light from the LCD screens, and a small amount of light from another workstation in the same room. Participants had different levels of experience at each workstation. The film workstation had been used by the participants for as long as they had been film reading, the hybrid workstation had been used by the participants for a period of two years prior to the commencement of the study. The digital workstation (i.e. of both current and prior mammograms displayed digitally) was introduced for the purposes of the study, and therefore participants had no experience of it.
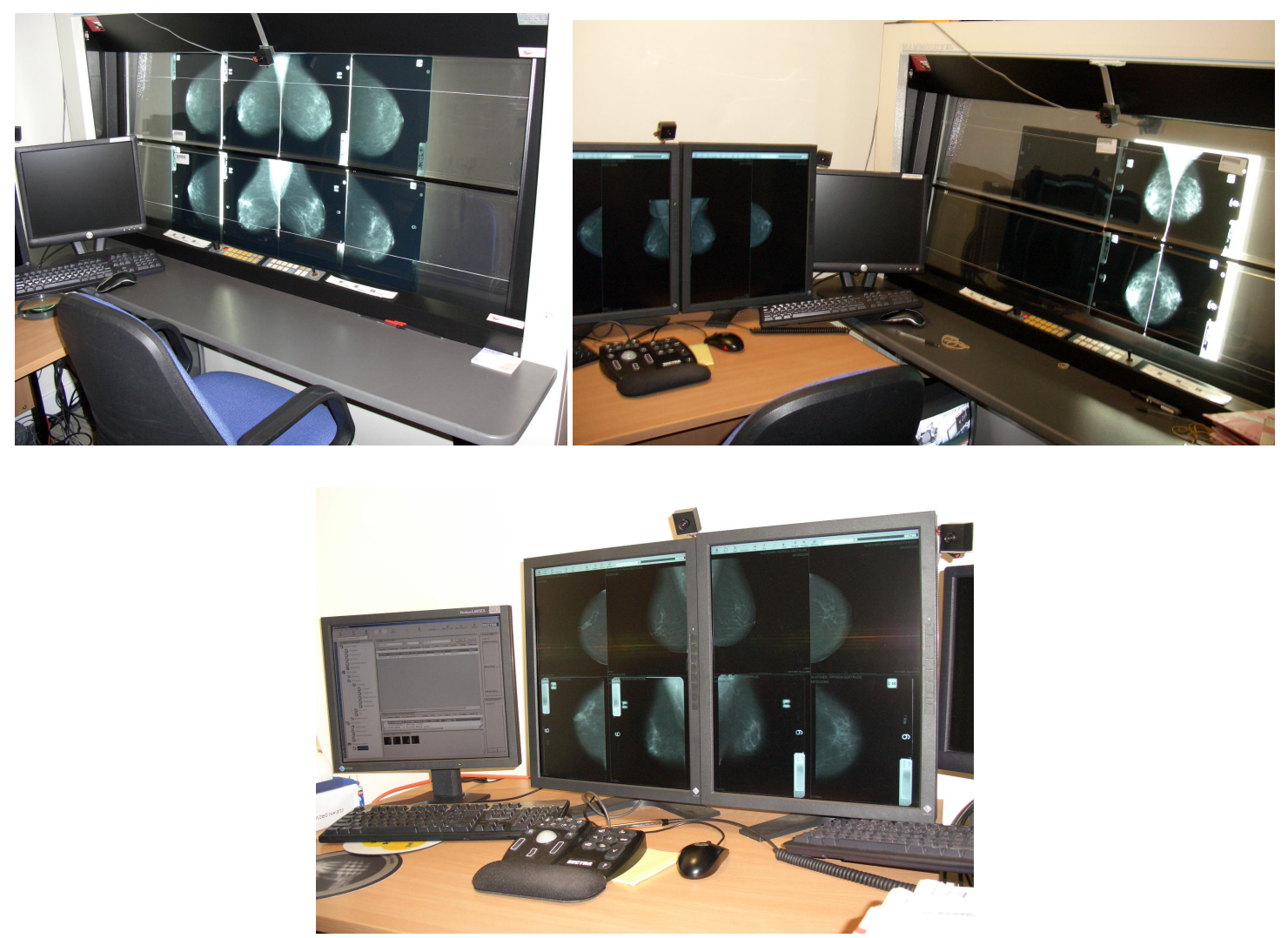

Figure 1 - A 'film workstation' (top left) with mammograms displayed on a multi-viewer. A 'hybrid workstation' (top right) with current mammograms displayed on the LCD screen and prior mammograms displayed on the multi-viewer. A 'digital workstation' (bottom) with current mammograms displayed on the upper half of the LCD screen and digitized prior mammograms displayed on the lower half

At the time of commencement of the study only two centres in the UK regularly used digital technology for breast screening, one of which was the Breast Screening Unit at University Hospitals Coventry and Warwickshire. All eight mammographers from that hospital were invited and agreed to take part, of which four were radiologists and four were radiographers. All were experienced film readers with experience ranging from 2.5 to 19 years, average eight years.

Each participant was video-taped from four different angles carrying out their routine reading practice for 45 minutes, filled out a body part discomfort questionnaire both before and after each session, and a NASA TLX workload questionnaire ${ }^{13}$ after each session. Each participant took part in two sessions at each of the three workstations. 
It was intended to change the order in which participants undertook the experiments at each of the three workstations using a Latin square design, but due to delays in the digitisation process all measurements at the digital workstation were taken after those at the film and hybrid workstations.

\subsection{Workstation Ergonomics}

To ascertain the levels of comfort at each workstation, and risk of radiologists developing musculoskeletal disorders after prolonged use, postural analysis was conducted in conjunction with body part discomfort charts for each session. Discomfort was rated for twelve body parts on a scale as follows: $1=$ no discomfort; $2=$ very mild discomfort; $3=$ mild discomfort; 4=moderate discomfort; and $5=$ severe discomfort. The Wilcoxon test was applied to changes of discomfort over the 45 minute reading session at each workstation, and the Friedman test applied to the differences between workstations. Data were collected for postural analysis using four cameras each at 90 degrees to one another surrounding the participant, these four images were synchronised and displayed together. Event based analysis was conducted on the films of each of the three workstations using the Rapid Upper Limb Assessment (RULA) ${ }^{14}$ postural analysis tool. Event based, rather than time based analysis was used to enable direct comparisons between the workstations whilst minimising the data points required. This is made possible as the same activities are conducted at all of the workstations. To determine the events to analyse a task analysis was conducted from initial short unstructured interviews with all participants, watching the films, and then additional questions to participants where necessary. Information in the academic literature was sought to determine how many repeat measurements were necessary to produce each data point for analysis, i.e. how many measurements for each event for each participant at each workstation, but as event based RULA is not a common method no direct precedent was found. It was decided that a minimum of three measurements at each workstation is necessary, at the beginning, middle, and end, so that any effects of fatigue can be recorded. Therefore, the events analysed were those closest in time to the following points, the earliest point in the timings, excluding the first case, as this may differ to the bulk of the cases analysed, 22.5 minutes through the 45 minute session, and the latest point excluding the last case. There are 17 events detailed, which would require analysis of 1,224 postures, which involves 9,792 data points. In order to focus analysis attention on the higher risk postures, only those actions which either occur more than four times per minute for a participant, involve reach in the extreme reach envelope (i.e. reaching that requires bending of the torso), or involve weights of greater than $2 \mathrm{kgs}$ were considered for analysis, leaving nine events. For those events which occurred over a finite time period, for example looking at the current mammogram, the most extreme posture within that time period was chosen. The Friedman test was applied to the differences in RULA score for each event between the three workstations, with Friedman post hoc tests where appropriate.

\subsection{Use of Prior Mammograms}

The number of comparisons to the prior mammogram was recorded using visual analysis of eye and head movements from the video data. A preliminary trial was conducted where seven participants who are not medical professionals sat at a multi-viewer workstation of similar design to that used in the study. These participants were asked to look at each of the eight possible positions in which the current mammograms could be displayed, and the eight possible positions the prior mammograms could be displayed whilst filming from a choice of two sets of camera locations. It was found that it was straightforward to distinguish between when participants were looking at current versus prior mammograms due to the consistent hanging protocol used at the trial hospital of current mammograms on the top row and corresponding prior mammograms on the row below. This same hanging protocol was applied to the digital workstation. An 'a priori' students t-test was conducted to analyse the difference between the mean number of comparisons per case at the hybrid and digital workstations. A one way ANOVA was carried out for average number of comparisons per case for all three workstations followed by pairwise post hoc tests with a Sidak adjustment for multiple comparisons.

\subsection{Workload}

After each session participants were asked to rate the six aspects of workload as defined by the NASA TLX questionnaire. A $100 \mathrm{~mm}$ long scale was marked for each question. The Friedman test was applied to the average marks at each workstation, followed by the Friedman post hoc test. 


\section{RESULTS}

\subsection{Workstation Ergonomics}

The only body area where there was an increase in reported discomfort during the reading session was the eyes at the film workstation $(\mathrm{p}=0.04)$. There was also a trend towards an increase in discomfort of the eyes at the hybrid workstation $(\mathrm{p}=0.07)$, and at the digital workstation $(\mathrm{p}=0.08)$. The difference between the increases in discomfort at the different workstations was not significant for any body area. Figure 2 shows the mean changes in discomfort by body area.

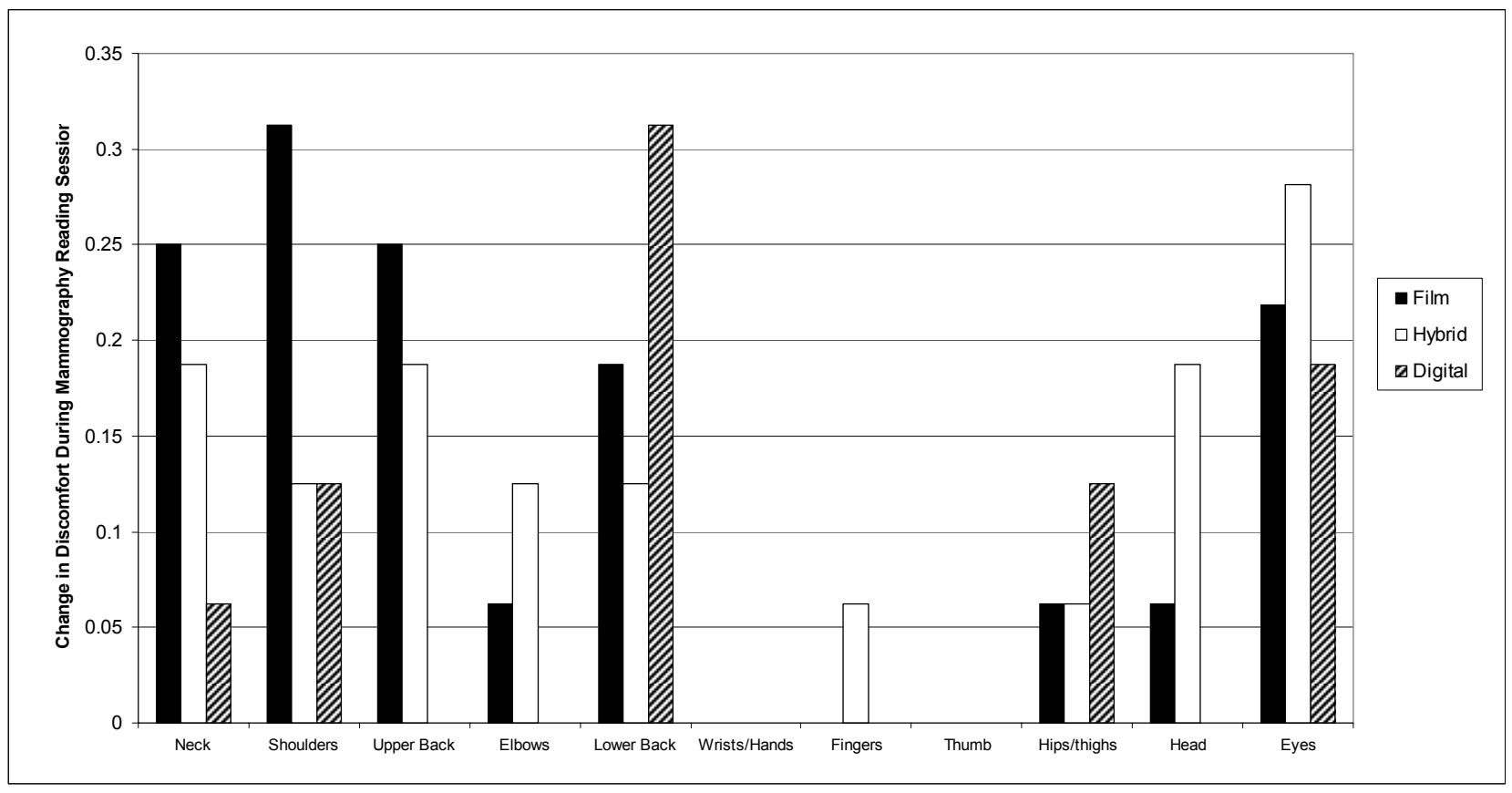

Figure 2: Average change in discomfort over the 45 minute mammography session. Scale of measurement was 1=no discomfort, $2=$ very mild discomfort, $3=$ mild discomfort, $4=$ =moderate discomfort, $5=$ severe discomfort.

The most frequent actions carried out were looking at the current and prior mammograms. At the film workstation looking at the current mammogram resulted in flexion of the neck due to the height at which the mammograms were hung, whereas this was not a problem when looking at the prior mammograms hung on the lower row. Therefore postural analysis showed a lower risk level when looking at the prior mammograms versus the current mammograms at the film workstation $(T=0, p=.04)$. This difference was not apparent at the digital workstation because the mammograms when all displayed together are both lower and smaller than at the film workstation (figure 1). This provides its own problems as mammographers tend to lean a long way forward over the desk to look more closely at the mammograms on the LCD screen. Results of the postural analysis are shown in figure 3. 


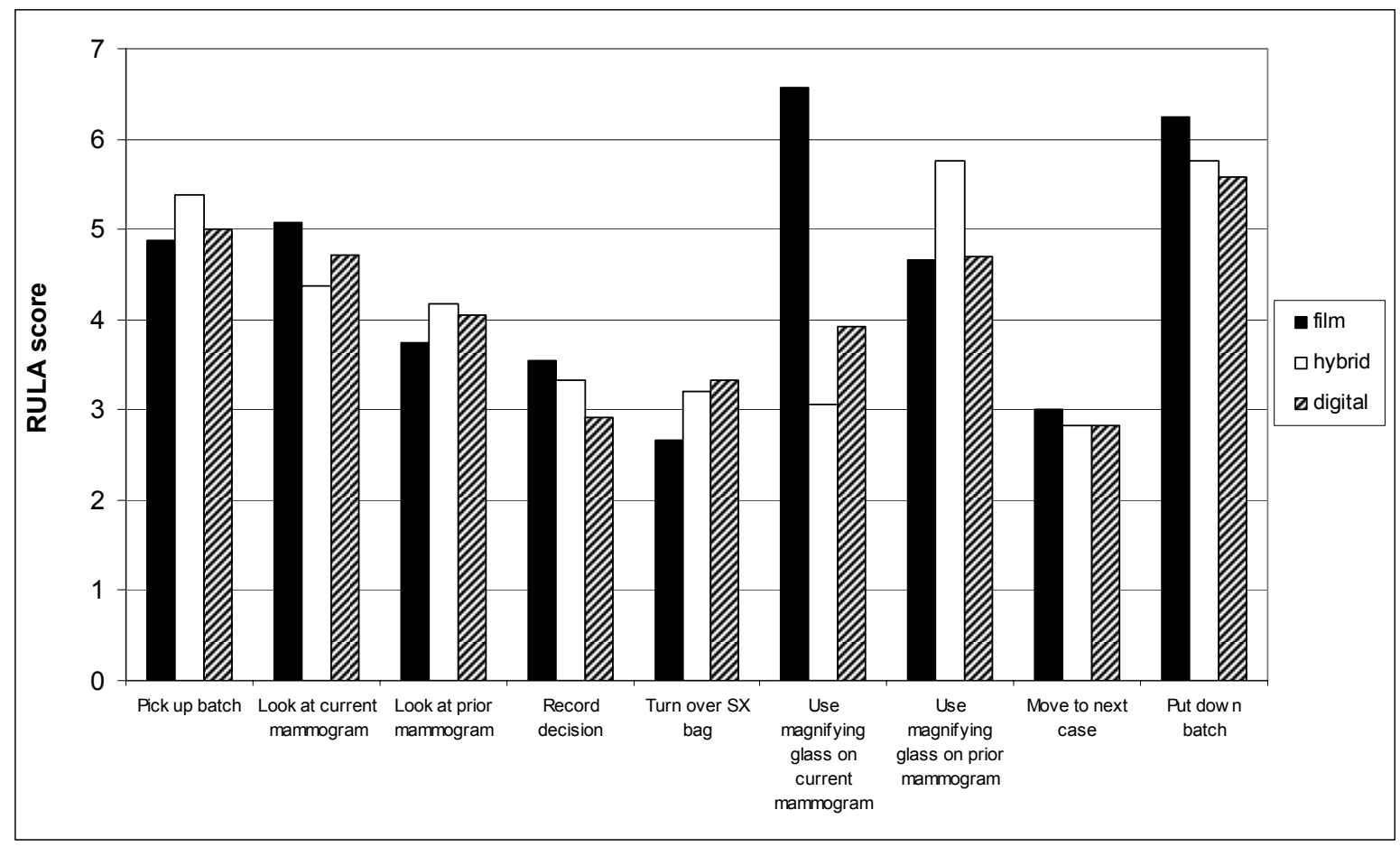

Figure 3 - Results of RULA postural analysis. A score of 1 or 2 indicates that posture is acceptable if it is not maintained or repeated for long periods, 3 or 4 indicates that further investigation is needed and changes may be required, 5 or 6 indicates that investigation and changes are required soon. A score of 7 indicates that investigation and changes are required immediately.

When using a magnifying glass to look at the current mammograms there was a difference between risk levels at the different workstations $\left(\chi^{2}(2)=10.2, p=.006\right)$, with the Friedman post hoc test showing higher risk postures were adopted at the film workstation in comparison to both the hybrid $(p<.05)$ and the digital workstation $(p<.05)$. This is due to a combination of flexion of the neck, leaning the torso left and right to view the mammograms on the end, and lifting a $500 \mathrm{~g}$ magnifying glass (figure 4).
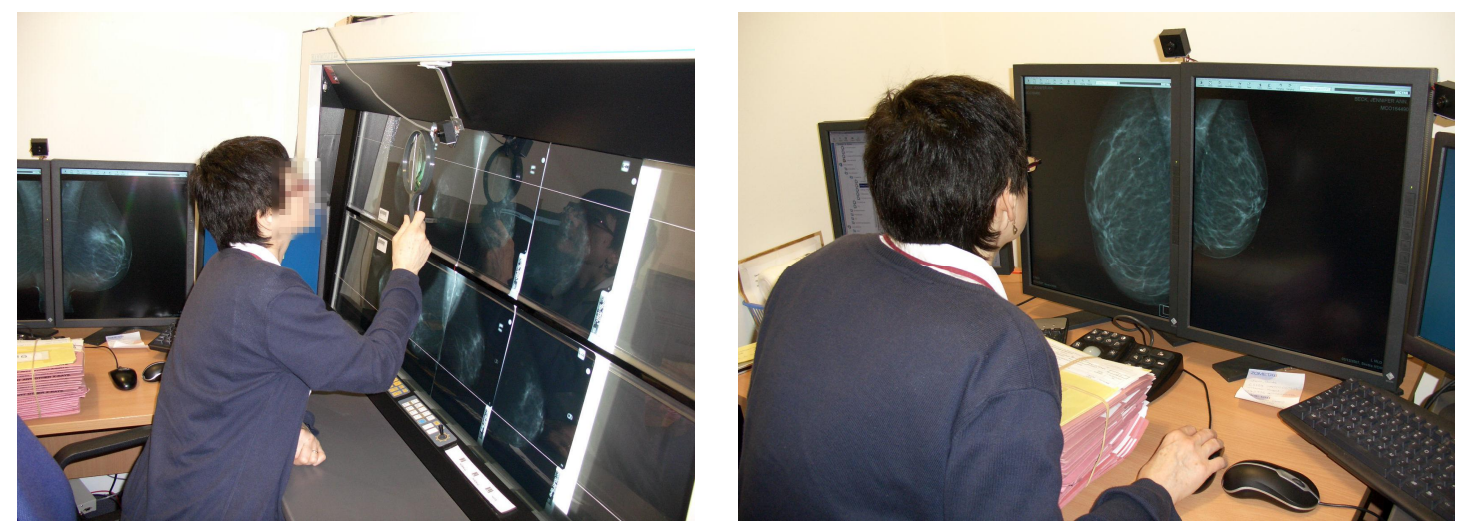

Figure 4 - A mammographer using magnification on the current mammograms at the film workstation (left) and the digital workstation (right) 
There was a problem of lack of desk space at the digital workstation, due to a combination of the aforementioned tendency to lean over the desk to get close to the LCD screen, alongside the space required for keyboard, mouse, and Sectra input keyboard. This resulted in little space for the ' $\mathrm{SX}$ ' bags containing the patient records and X-ray films (figure 5), and consequently a difference in postural risk level between the workstations for turning over the SX bags to move to the next case $\left(\chi^{2}(2)=9.2, p=.01\right)$. Friedman post hoc tests showed a trend towards higher postural risk at the digital workstation in comparison to the film workstation (difference in ranks $=1.19$, critical difference $=1.2$ ) due to mammographers' coping strategies in dealing with this lack of space, which involved stretching to place the SX bags in a range of different locations. This would not be an issue if a full paperless reporting system were established.
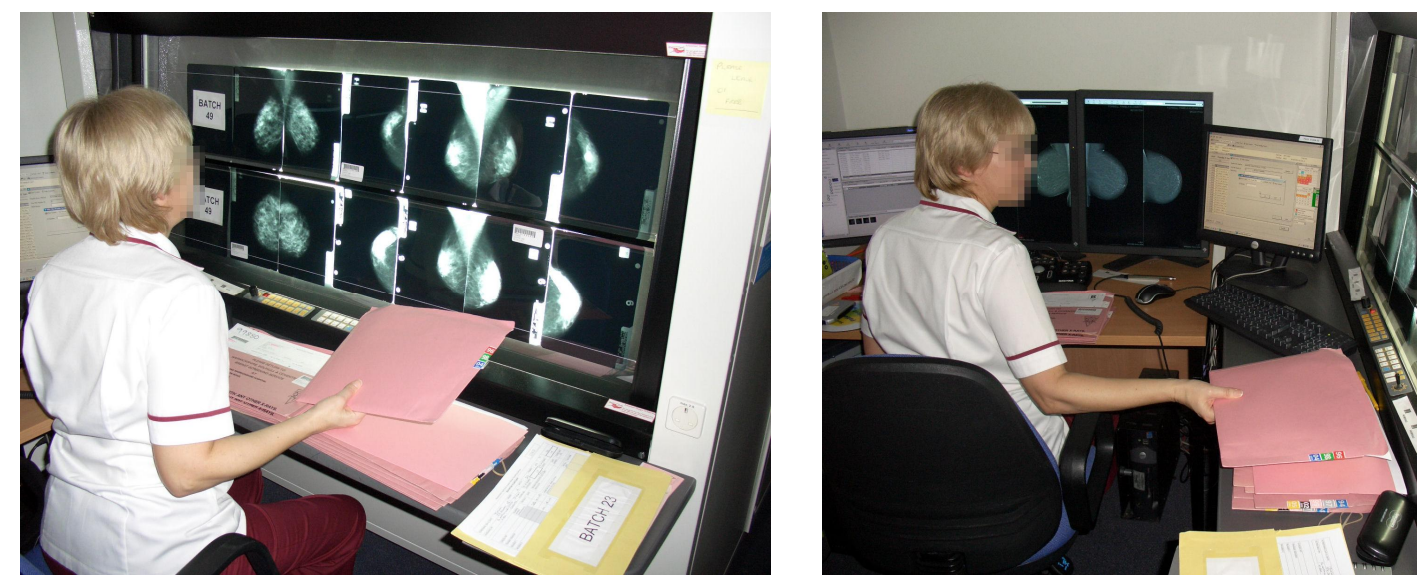

Figure 5 - A mammographer turning over an SX bag to move to the next case at the film workstation (left) and the digital workstation (right).

\subsection{Use of Prior Mammograms}

The average number of glances at the prior mammograms was greater at the digital versus the hybrid workstation $(t(7)=-2.65, p=.03)$. Therefore during the transition period to digital mammography if the prior mammograms are digitised the mammographers will look at them a greater number of times per case. A one way analysis of variance showed differences between the three workstations $(F(2,14)=9.3, p=.003)$, with pairwise post hoc comparisons using a Sidak correction demonstrating more glances at the film than the hybrid workstation $(p=0.02)$. Therefore, if the decision is taken not to digitise the prior mammograms during the transition period, then the implication is that mammographers will look at the prior film mammograms fewer times per case than they were before the introduction of digital mammography.

\subsection{Workload}

There was a difference between the three workstations for un-weighted overall NASA TLX workload $\left(\chi^{2}(2)=6.3 p=.04\right)$. Friedman post hoc analysis showed that overall workload was higher at the hybrid than the digital workstation $(p<.05)$. Mental demand was recorded as a sub-scale of overall workload, and was found to differ depending on the workstation used $\left(\chi^{2}(2)=7.0 p=.03\right)$. The Friedman post hoc test showed a higher mental demand at the hybrid than the digital workstation $(p<0.05)$.

At the end of the experiment participants were asked to rank the workstations in order of preference. Seven out of the eight participants preferred the digital workstation to both the hybrid and the film workstations. 


\section{DISCUSSION}

No evidence was found to suggest that discomfort or risk of musculoskeletal disorders will increase either during or after the transition to digital mammography. However, this cannot be considered conclusive evidence due to the small sample size. Body part discomfort charts showed no increases in discomfort over a 45 minute reporting session in any body part, apart from the eyes, at any of the workstations. This is encouraging because mammography reading is often undertaken over short sessions in the UK. Postural analysis has shown that for the most common activities in film reading there will not be an overall increase in risk factors associated with the postures adopted, but there will of course be changes to those postures which will increase pressures on certain body areas whilst relieving it in others. For the activity of looking at the current mammogram with magnification the overall posture of the mammographer will improve with the introduction of digital mammography, as the current mammograms will be displayed at a lower height so reducing flexion in the neck, and the magnification will be electronic so there is no need to hold a magnifying glass. However, flexion of the back may be increased as mammographers' lean closer to the LCD screen.

The data collected here suggest that when designing digital mammography workstations several aspects must be carefully considered. Firstly, that when needing a magnified view the natural instinct is to move the head closer to the image, before considering using a magnification tool. Therefore, this should be possible without adopting extreme postures. However, it is preferable that mammographers use the digital magnification tool, as using external magnification will not change the finite pixel size of the VDU screen, and moving the head close to the VDU screen has been associated with eye $\operatorname{strain}^{15,16}$. Therefore, magnification tools should be simple and intuitive to operate, and as similar to the known activity of using a magnifying glass as possible. Finally, the height of the VDU screens must be low enough so that the shortest mammographer using the workstation does not have to put their neck into flexion to look at the current mammograms.

If the prior mammograms are not digitised then during the three year transition to digital mammography this study indicates that mammographers will look at them fewer times per case than if they are digitised, and furthermore mammographers will look at them fewer times than they did previously when reading film mammograms. There are two possible explanations for this behaviour: Firstly, that the mammographers are making less use of the prior mammograms in their decision making. If this is the case then unless prior mammograms are digitised the performance of mammographers will potentially decrease during the three year transition, and furthermore it may impact their mammography reading behaviour beyond the three year transition period. The second possibility is that the mammographers are taking fewer glances, but taking in more information per glance at the hybrid workstation. The implications of this are more difficult to predict. This could involve an increase in mammographers' use of short term memory when making comparisons between current and prior mammograms, or it could represent some change in the methodology the mammographer is using to interpret the mammograms. However, it is clear that there is a change in observed mammographer behaviour, which may have implications for performance.

There was higher perceived mental demand and overall workload at the hybrid workstation than at the digital workstation. There could be many different reasons for this but the three that appear most likely are as follows: The mammographers could be looking at the prior mammograms less, but storing more information per glance which would increase mental demand and workload. There could be increased mental demand in switching constantly between the different appearances of the digital mammograms displayed on LCD screen and prior film mammograms displayed on the multi-viewer. It could simply be due to the extra effort required at the hybrid workstation to keep the multi-viewer and the digital workstation on the same case as one another. The implication of this finding in the transition to widespread full digital mammographic screening is that by digitising the prior mammograms then the perceived workload of mammographers could be reduced. In the context of an increasing screening population, with concomitant possible shortages of experienced mammographers, then this would be a very valuable benefit for mammographers whose current workload is already high.

\section{ACKNOWLEDGEMENT}

We gratefully acknowledge the contribution of the participants and staff at the Breast Screening Unit at University Hospitals Coventry and Warwickshire who gave up their time to assist with this research. 


\section{REFERENCES}

[1] Thurfjell, M. G., Vitak, B., Azavedo, E., Svane, G., Thurfjell, E., "Effect on Sensitivity and Specificity of Mammography Screening with or Without Comparison of Old Mammograms", Acta Radiol., 41(1), $52-56$ (2000).

[2] Sumkin, J. H., Holbert, B. L., Herrmann, J. S., Hakim, C. A., Ganott, M. A., Poller, W. R., Shah, R., Hardesty, L. A., Gur, D. , "Optimal reference mammography: a comparison of mammograms obtained 1 and 2 years before the present examination", Am. J. Roentgenol., 180(2), 343-346 (2003).

[3] Varela, C., Karssemeijer, N., Hendriks, J. H. C. L., Holland, R., "Use of prior mammograms in the classification of benign and malignant masses", European Journal of Radiology, 56(2), 248-255 (2005).

[4] Roelofs, A. A., Karssemeijer, N., Wedekind, N., Beck, C., van Woudenberg, S., Snoeren, P. R., Hendriks, J. H., Rosselli del Turco, M., Bjurstam, N., Junkermann, H., Beijerinck, D., Seradour, B., Evertsz, C. J. , "Importance of comparison of current and prior mammograms in breast cancer screening", Radiology, 242(1), 70-77 (2007).

[5] Gilbert, F. J., Astley, S. M., McGee, M. A., Gillan, M. G., Boggis, C. R., Griffiths, P. M., Duffy, S. W. , "Single reading with computer-aided detection and double reading of screening mammograms in the United Kingdom National Breast Screening Program", Radiology, 241(1), 47-53 (2006).

[6] Patnick,, J., "NHS Breast Screening Programme Annual Review 2007", Hanover, Sheffield, 5 (2007).

[7] Karssemeijer, N., personal communication, (2008).

[8] Hill, S. J., Faulkner, K., Law, J., Starritt, H. C. , "Film viewing conditions in mammography", Br. J. Radiol., 70(832), 409-411 (1997).

[9] Kimme-Smith, C., Haus, A. G., DeBruhl, N., Bassett, L. W., "Effects of ambient light and view box luminance on the detection of calcifications in mammography", Am. J. Roentgenol., 168(3), 775-778 (1997).

[10] Horii, S. C., Horii, H. N., Mun, S. K., Benson, H. R., Zeman, R. K. , "Environmental Designs for Reading from Imaging Workstations: Ergonomic and Architectural Features", J. Digital Imaging, 16(1), 124-131 (2003).

[11] Nagy, P., Siegel, E., Hanson, T., Kreiner, L., Johnson, K., Reiner, B., "PACS reading room design", Semin. Roentgenol., 38(3), 244-255 (2003).

[12] Ratib, O., Valentino, D. J., McCoy, M. J., Balbona, J. A., Amato, C. L., Boots, K. , "Computer-aided design and modeling of workstations and radiology reading rooms for the new millennium", Radiographics, 20(6), 1807-1816 (2000).

[13] Hart, S. G., Staveland, L. E., "Development of NASA-TLX (Task Load Index): Results of empirical and theoretical research", Hancock, P. A., Meshkati, N., Eds.; [Human mental workload], North-Holland, Oxford, England, 139$183,(1988)$.

[14] McAtamney, L., Corlett, E. N. , "RULA: a survey method for the investigation of work-related upper limb disorders", Appl. Ergon., 24(2), 91-99 (1993).

[15] Owens, D. A., Wolf-Kelly, K., "Near work, visual fatigue, and variations of oculomotor tonus", Invest. Ophthalmol. Vis. Sci., 28(4), 743-749 (1987).

[16] Jaschinski-Kruza, W., "Eyestrain in VDU users: viewing distance and the resting position of ocular muscles", Hum. Factors, 33(1), 69-83 (1991). 